\title{
Hemodynamic Determinants of the Peak Systolic Left Ventricular-Aortic Pressure Gradient in Children with Valvar Aortic Stenosis
}

Robert H. Beekman, MD, Albert P. Rocchini, MD, Joseph H. Gillon, and G. B. John Mancini, MD

$\mathbf{T}$ he peak systolic pressure gradient (the difference between peak left ventricular [LV] and peak aortic systolic pressures) has been used for many years as a primary measure of severity in children with valvar aortic stenosis (AS) ${ }^{1-3}$ Reliance on the peak systolic pressure gradient in clinical decision-making was based on the practice of measuring the gradient at catheterization by withdrawing a single catheter from the left ventricle to aorta. In recent years it has become common, however, to measure LV and aortic pressures simultaneously with dual catheter techniques. ${ }^{4}$ The time-honored peak systolic gradient does not actually exist in time, because peak LV pressure in AS occurs well before peak aortic systolic pressure. The pressure gradients that actually exist in real time between the left ventricle and aorta (the instantaneous gradients) vary throughout systole. These instantaneous pressure gradients, which include peak instantaneous gradient and mean systolic gradient (the integral of the systolic instantaneous gradients) can be estimated noninvasively, ${ }^{5-7}$ unlike peak systolic gradient. Nevertheless, clinicians continue to rely on peak systolic pressure gradient as an important index of severity for clinical decision-making in children with valvar AS. The purpose of this study was to define, at cardiac catheterization, the relation between peak, mean and peak instantaneous systolic pressure gradients in children with valvar AS.

The patient group consisted of 34 children and adolescents (aged 3 months to 24 years [mean $\pm S D 10.5 \pm$ 6.4 years, median 11]) with isolated valvar $A S$ who underwent cardiac catheterization at our institution between 1985 and 1990. Their weights ranged from 6.8 to $105 \mathrm{~kg}$ (mean 43.5 \pm 27.5 ). Peak systolic AS gradient at rest in these children ranged from 13 to $109 \mathrm{~mm} \mathrm{Hg}$, and the degree of aortic regurgitation ranged from 0 to $3+$ (on a 0 to $4+$ scale). Twenty children had native valvar $A S$ (i.e., no prior treatment) and 14 children had undergone prior balloon valvuloplasty. No children with prior surgical valvotomy were included in this study.

Cardiac catheterization was performed percutaneously after light sedation with morphine sulfate and chloral hydrate. Complete right - and left-sided cardiac catheterization was performed, and cardiac output was measured in triplicate using the thermodilution technique. The left-sided pressures were recorded using 2 identical 4 to 7 Fr pigtail catheters (UMI, Ballston Spa, New York) and fluid-filled transducers (Sorenson, No. Chicago, Illinois). In each patient both pigtail catheters were initially positioned side-by-side in the descending aorta to confirm that identical waveforms were recorded.

From the Division of Pediatric Cardiology, Department of Pediatrics, Box 0204, Room F1312, C.S. Mott Children's Hospital, University of Michigan Medical Center, Ann Arbor, Michigan 48109. Manuscript received September 12, 1991; revised manuscript received November 25,1991 , and accepted November 30
The left ventricle was then entered in a retrograde fashion and the pigtail catheter positioned in the LV apex. The aortic catheter was positioned in the ascending aor$t a$, so that the upstroke of the aortic waveform occurred immediately on crossing the LV pressure waveform (i.e., no time delay). Simultaneous $L V$ and ascending aortic pressure measurements were then recorded at a paper speed of 50 to $100 \mathrm{~mm} / \mathrm{s}$. All measurements were $o b$ tained with the patient in a steady-state condition, in sinus rhythm, and before angiography.

The simultaneous $L V$ and ascending aorta pressure tracing from each patient was digitized (SigmaScan Valve Analysis Program), and data from 2 to 3 consecutive beats were averaged. Data obtained from the digitized tracings (Figure 1) included the peak systolic, mean systolic, and peak instantaneous systolic gradients, aortic pulse pressure, systolic ejection period, $R R$ interval, time from $R$ wave to peak $L V$ pressure, and time from $R$ wave to peak aortic pressure. A stepwise multiple linear regression analysis was then performed with the peak systolic gradient as the dependent variable. Independent variables studied were the digitized pressure waveform variables previously noted, as well as $L V$ stroke volume and degree of aortic regurgitation. The stepwise multiple regression analysis was performed with an $F$ value of 4.0 required for a variable to enter the

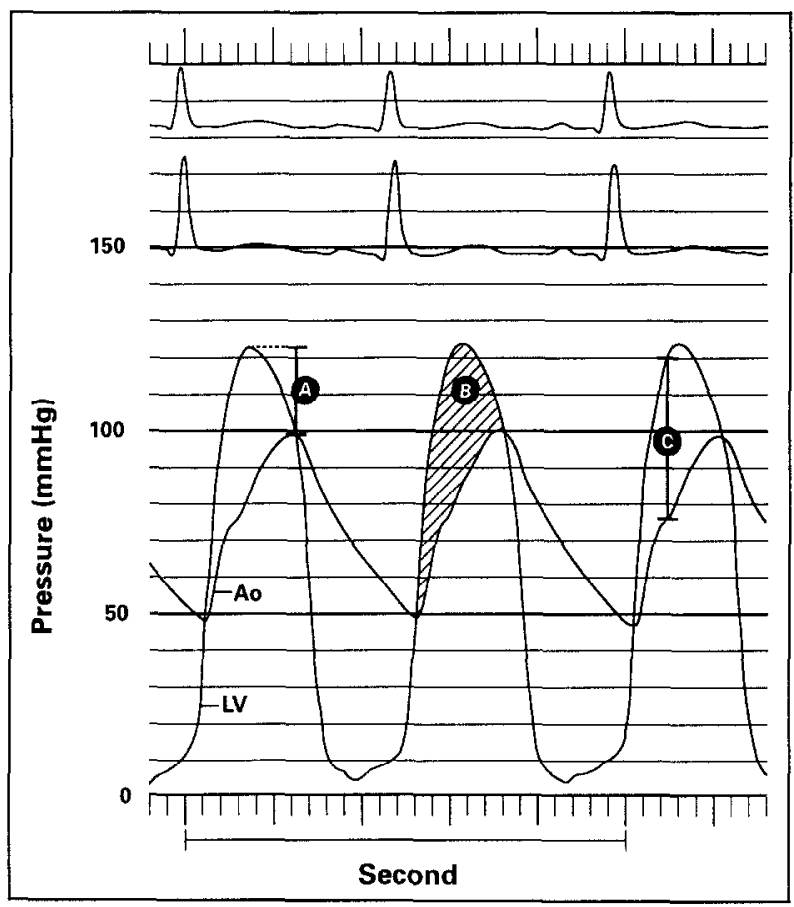

FIGURE 1. Simuttaneous left ventricular (LV) and aortic pressure (Ao) trachigs demonstrating the peak (A), mean (B) and peak instantaneous (C) systolic pressure gradients in a child with valvar aortic stenosis. 


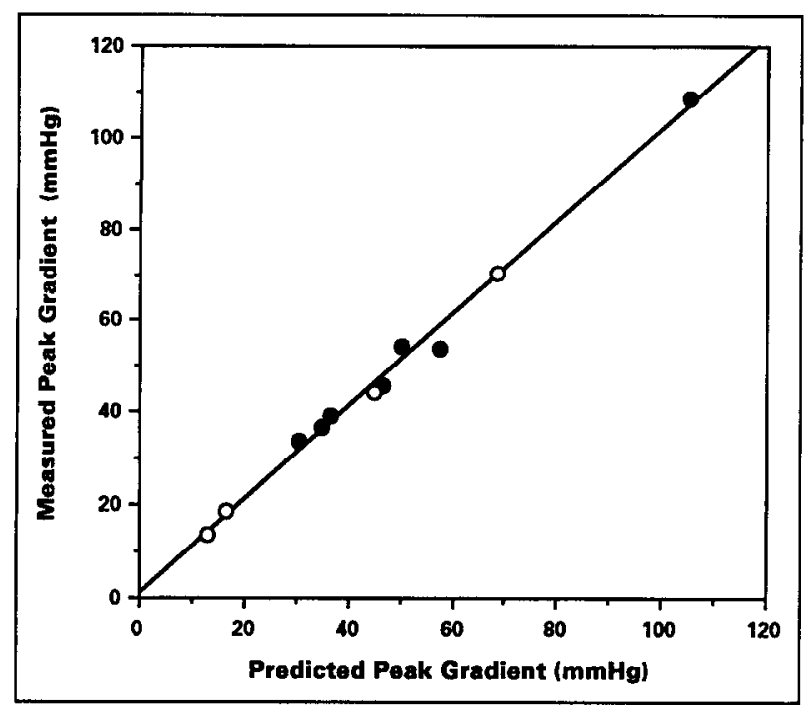

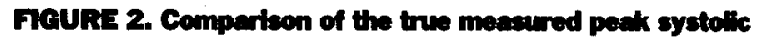
gradiont with that predicted by the model from the mean gra-

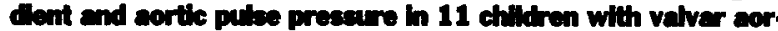
tic stensis. Data from these chiliren were not used to derive the prediction madel. Cloced afrales, nathe valves; open c/r

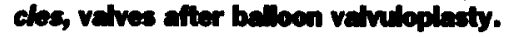

model, and an $F$ value of 3.95 to remove a variable from the model (StatView II, Abacus Concepts, Berkeley, California).

Using data from the 23 patients who had cardiac catheterization between July 1985 and July 1989, we defined a model to predict the peak systolic $A S$ pressure gradient. This model was then validated in a second group of 11 consecutive patients who underwent catheterization after July 1989, and whose data were not used in defining the model. In this validation study, the peak systolic gradients predicted by the model were compared by simple linear regression analysis with peak gradients measured by dual catheter technique at catheterization.

Stepwise multiple linear regression analysis identified the mean systolic gradient (mean $\Delta$ ) and the aortic pulse pressure as the most precise predictors of the peak systolic $A S$ pressure gradient (peak $\Delta$ ) in this patient population. The model is as follows $\left(F=356, R^{2}=0.97\right.$, $S E E=4.3 \mathrm{~mm} \mathrm{Hg})$ : peak $\Delta=6.02+1.49($ mean $\Delta)-$ 0.44 (pulse pressure). Thus, $97 \%$ of the variability in peak systolic $A S$ gradient could be explained by inclusion of only 2 independent variables in the model (the mean systolic gradient and the aortic pulse pressure). There was no further significant reduction in variance by inclusion in the equation of any additional study variables ( $F$ to enter equation: LV stroke volume 3.72; peak instantaneous gradient 3.36; all other variables <1.0).

To validate the model, peak systolic $A S$ pressure gradient was predicted from the mean gradient and aortic pulse pressure measured at cardiac catheterization in 11 consecutive children with valvar AS studied after July 1989. Data from these patients were not used to derive the model. In these 11 children, peak systolic gradient predicted from the model was compared with the peak systolic gradient measured directly by catheter (Figure 2). The relation between peak AS gradients predicted by the model and the true measured peak gradients nearly describes the line of identity $(Y=0.82+$ $1.01 X, F=1160, R^{2}=0.99$ ). Furthermore, the ability of the model to predict the measured peak gradient does not appear affected by whether the valve was untreated or had undergone prior valvuloplasty (Figure 2).

Using digitized pressure data obtained from fluidfilled catheters, we have found that the peak systolic pressure gradient can be accurately predicted from the mean systolic gradient and aortic pulse pressure in children with valvar AS. The model derived from data in 23 children was subsequently validated in 11 children with valvar AS, whose data were not used to derive the model. In the validation study, the model was found to be extremely accurate in predicting the true peak systolic gradient from the measured mean gradient and aortic pulse pressure (Figure 2). Inclusion of additional variables (including the peak instantaneous gradient) did not significantly improve the model.

It is not entirely unexpected that mean systolic gradient and aortic pulse pressure relate closely to peak systolic gradient in children with valvar AS. Mean systolic gradient is affected not only by the degree of anatomic valve

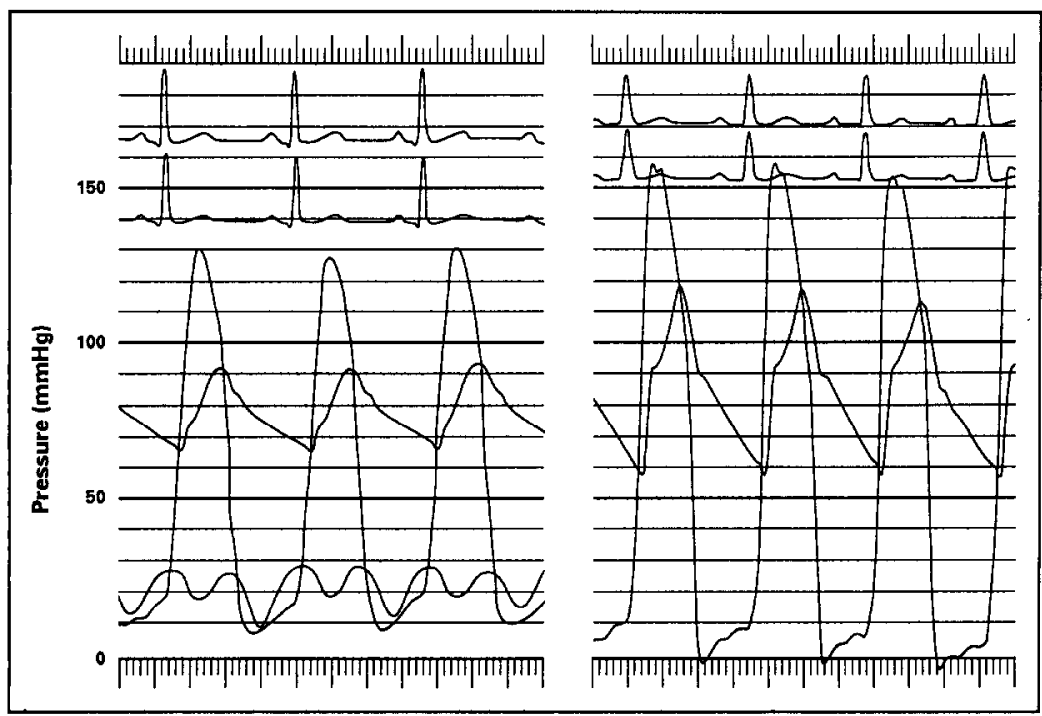

FIGURE 3. Prescure trachess from 2 ch"ldren with valvar aortic stenods and equal pask systelie gradiente of approximately $40 \mathrm{~mm} \mathrm{Hg}$. The much lerger aortic pulse presecure explalns the grealer mean and peak instantaneous gradients in the patemt on the right panel. Left panekt peak gradient, $38 \mathrm{~mm}$ Hg; pulse pressure, 28 mm Hss mean gradent, $28 \mathrm{~mm}$ Hst peak instantaneous gradient, $45 \mathrm{~mm} \mathrm{Hg}$. Plght panels peak gradient, $40 \mathrm{~mm}$ Hz; putbe prescure, $62 \mathrm{~mm}$ Hs; mean gradient, 42 mm Hg; peak Instantanoous gradient, 67 mm Hs. 
deformity, but also by the magnitude of transvalvar blood flow (in turn related to LV stroke volume and systolic function). Thus, mean systolic gradient brings to the model information regarding the patient's valve stenosis and hemodynamic status. With the mean gradient in the model no additional significant information was added by inclusion of cardiac output, stroke volume, RR interval, systolic ejection period or the peak instantaneous gradient. These observations are consistent with a recent retrospective report by Bengur et $\mathrm{al}^{8}$ who noted that Doppler mean systolic gradient was the best noninvasive predictor of need for intervention in children with valvar AS. Our data explain this finding since the mean gradient has been shown to relate closely to the peak systolic gradient which, in turn, has been the primary basis for clinical management decisions in these patients.

The model derived in the present study indicates that the aortic pulse pressure also plays an important role in determining peak systolic gradient in children with valvar AS. Aortic pulse pressure is affected by severity of valve stenosis, degree of aortic regurgitation, LV stroke volume and peripheral vascular resistance. Information pertaining to these hemodynamic factors is contributed to the model by aortic pulse pressure data. Figure 3 illustrates the importance of aortic pulse pressure in explaining relations between pressure gradients in children with AS. As illustrated, 2 children with nearly identical peak systolic gradients may have widely discrepant mean gradients (and peak instantaneous gradients) if aortic pulse pressures differ substantially. Conversely, for a given mean systolic gradient, peak systolic gradient will be lower if a patient's aortic pulse pressure is greater (as explained by the model: peak $\Delta=6.02+1.49[$ mean $\Delta]-0.44$ [pulse pressure]).

1. Friedman WF, Modlinger J, Morgan J. Serial hemodynamic observations in asymptomatic children with valvar aortic stenosis. Circulation 1971;43:91-97. 2. Wagner HR, Ellison RC, Keane JF, Humphries JO, Nadas AS. Clinical course in aortic stenosis. Circulation 1977;56(suppl I):I-47-I-57.

3. Wagner HR, Weidman WH, Ellison RC, Miettinen OS. Indirect assessment of severity in aortic stenosis. Circulation 1977;56(suppl I):I-20-I-23.

4. O'Connor BK, Beekman RH, Rocchini AP, Rosenthal A. Intermediate-term effectiveness of balloon valvuloplasty for congenital aortic stenosis: a prospective follow-up study. Circulation 1991;84:732-738.

5. Hatle L, Angelsen BA, Tromsdal A. Non-invasive assessment of aortic stenosis by Doppler ultrasound. $B r$ Heart $J$ 1980;43:284-292.

6. Oliveira LC, Sahn DJ, Valdes-Cruz LM, Allen HD, Goldberg SJ, Grenadier E, Barron JV. Prediction of the severity of left ventricular outflow tract obstruction by quantitative two-dimensional echocardiographic Doppler studies. Circulation $1983 ; 68: 348-354$

7. Currie PJ, Hagler DJ, Seward JB, Reeder GS, Fyfe DA, Bove AA, Tajik AJ. Instantaneous pressure gradient: a simultaneous Doppler and dual catheter correlative study. I Am Coll Cardiol 1986;7:800-806.

8. Bengur AR, Snider AR, Serwer GA, Peters J, Rosenthal A. Usefulness of the Doppler mean gradient in evaluation of children with aortic valve stenosis and comparison to gradient at catheterization. Am I Cardiol 1989;64:756-761.

\title{
Percutaneous Closure of the Small $(<2.5 \mathrm{~mm}$ ) Patent Ductus Arteriosus Using Coil Embolization
}

\author{
Patrick A. Cambier, MD, William C. Kirby, MD, Dale C. Wortham, MD, and John W. Moore, MD
}

$\mathbf{P}$ ersistently patent ductus arteriosus (PDA) is a common form of congenital heart disease, occurring between 0.01 to $0.08 \%$ of live births. ${ }^{1}$ Surgical ligation by lateral thoracotomy is effective, yet carries procedural risks (potential bleeding, recurrent laryngeal nerve injury, exposure to general anesthesia and death $[<1 \%]) .{ }^{2}$ In 1971 , Porstmann et $\mathrm{al}^{3}$ described the first nonsurgical PDA closure with an Ivalon plug with an $18 \mathrm{Fr}$ arterial conduit. More recently, the Rashkind PDA double disc occlusion system has undergone extensive evaluation, with flow occlusion accomplished in 72 to $88 \%$ of cases attempted..$^{4-6}$ The smaller Rashkind occluder requires an 8 Fr delivery system. Rao et $\mathrm{al}^{7}$ described an occluder which may be introduced through a $7 \mathrm{Fr}$ catheter and reduces the crossing profile required. Nevertheless, technical limitations persist in patients whose PDA internal diameter is $<2.5 \mathrm{~mm}$.

Techniques to facilitate cannulation of the very small ductus by the Rashkind delivery system, involving arterial cannulation and rendezvous of an exchange wire from femoral vein to contralateral artery, ${ }^{8}$ and ductal dilation with balloon angioplasty, ${ }^{9}$ have been described. These techniques require additional manipulations and procedural time, and may potentially increase patient morbidity. This report describes experience with an alternative

From the Cardiology Service, Walter Reed Army Medical Center, Washington, D.C. 20307-5001. Manuscript received October 15, 1991; revised manuscript received November 26, 1991, and accepted November 30 . technique for transcatheter closure of the very small ductus arteriosus using stainless steel coil embolization.

Between June 1990 and February 1991, 4 patients were diagnosed with a very small persistently patent ductus, whose parents had requested a nonsurgical closure. The diagnosis was made by clinical examination, 2-dimensional and color flow Doppler. PDA internal diameter was estimated by means of color flow characteristics. Coil embolization as a means of transcatheter closure was discussed and informed consent obtained in accordance with institutional policy.

Patients received ketamine sedation at appropriate dosage for weight. Cefazolin, $60 \mathrm{mg} / \mathrm{kg}$, was administered intravenously. A $5 \mathrm{Fr}$ sheath was placed into the femoral vein, and routine right heart pressures and oxy. gen saturations were obtained. A 5 Fr sheath was placed into the femoral artery and a pigtail catheter situated proximal to the PDA aortic diverticulum. Heparin, 100 $U / k g$, was administered. Aortography was performed in the anterior and $90^{\circ}$ lateral projections (Figure l). The pigtail catheter was exchanged for a 5Fr Judkins no. 4 angiographic catheter which was placed into the aortic diverticulum. Hand injections of contrast permitted $P D A$ size determination. Specific internal diameter measurements were obtained by use of magnification correction using reference comparison with known angiographic catheter dimension. The Judkins catheter was hand secured in the aortic diverticulum while a 0.038 inch (wire diameter), $3 \mathrm{~mm}$ (helical diameter) $\times 40 \mathrm{~mm}$ 